\title{
Intraoperative evaluation using mobile computed tomography in anterior cervical decompression with floating method for massive ossification of the posterior longitudinal ligament
}

Toshitaka Yoshii ${ }^{1,2,4^{*}}$, Takashi Hirai ${ }^{1,2,4}$, Tsuyoshi Yamada ${ }^{1,2,4}$, Hiroyuki Inose ${ }^{1,4}$, Tsuyoshi Kato ${ }^{1,4}$, Kenichiro Sakai ${ }^{3,4}$, Mitsuhiro Enomoto ${ }^{1,2,4}$, Shigenori Kawabata ${ }^{1,4}$, Yoshiyasu Arai ${ }^{3,4}$ and Atsushi Okawa $a^{1,2,4}$

\begin{abstract}
Background: An anterior decompression and fusion (ADF) with the floating method is an effective procedure for treating ossification of the posterior longitudinal ligament (OPLL), allowing a direct decompressive effect on the spinal cord. However, the procedure is skill-intensive, particularly in cases of OPLL with a high canal-occupying ratio. In such cases, there are potential risks for insufficient decompression due to the incomplete floating of the OPLL. Here, we introduce an anterior decompression procedure for massive OPLL, using an intraoperative computed tomography (CT) with a mobile scanner gantry for the intraoperative evaluation of the decompression. We further evaluated the outcomes of ADF using mobile CT in comparison with a historical control of ADF without intraoperative CT evaluation.
\end{abstract}

Methods: Fifty OPLL patients who underwent ADF with the floating method were evaluated in this study: 25 patients with intraoperative $C T$ (CT group) and 25 patients without $C T$ (non- $C$ group). In the $C T$ group, intraoperative $C T$ scanning was performed before freeing the ossification from the surrounding bone tissues. The reconstructed images were reviewed to evaluate the extent of bone decompression and thinning of the OPLL. After review of the images, further thinning of the OPLL or removal of surrounding bone was performed as deemed necessary, to complete the floating of the OPLL.

Results: Patients' background was similar between the $C T$ and non- $C T$ group. Operating time tended to be shorter for the $C T$ group. On the postoperative $C T$, incomplete OPLL floating due to "impingement" between the OPLL and the medial aspect of the pedicle or uncovertebral joint was observed for four patients (16.0\%) in the non-CT group, whereas insufficient decompression was not observed in the CT group.

Conclusions: Intraoperative CT imaging was effective to avoid insufficient decompression following ADF with the floating method for massive OPLL. We also consider that the intraoperative three-dimensional imaging is helpful for providing informative feedback to surgeons to improve performance in skill-intensive surgeries such as ADF with the floating method.

Keywords: Ossification of the posterior longitudinal ligament (OPLL), Anterior decompression, Floating method, Computed tomography $(C T)$, Intraoperative evaluation

\footnotetext{
* Correspondence: yoshii.orth@tmd.ac.jp

${ }^{1}$ Department of Orthopaedic Surgery, Graduate School, Tokyo Medical and

Dental University, 1-5-45 Yushima, Bunkyo-ku, Tokyo 113-8519, Japan

${ }^{2}$ Section of Regenerative Therapeutics for Spine and Spinal Cord, Graduate

School, Tokyo Medical and Dental University, 1-5-45 Yushima, Bunkyo-ku,

Tokyo 113-8519, Japan

Full list of author information is available at the end of the article
} 


\section{Background}

Cervical ossification of the posterior longitudinal ligament (OPLL) is a common degenerative spinal disease that causes neurological dysfunction $[1,2]$. In the early stages, the majority of patients with OPLL may not exhibit any neurological symptoms. As OPLL develops in the spinal canal, the spinal cord and nerve roots are compressed from the anterior direction, which results in myelopathy and/or radiculopathy. Minimally symptomatic patients may be treated conservatively; however, patients with progressive neurological disturbances require surgical treatment $[3,4]$.

The choice of the optimal surgical procedure for the treatment of cervical OPLL is controversial. Posterior decompression, such as laminoplasty, is relatively simple and has a low complication rate [5]. However, the effect of indirect decompression of the spinal cord is limited for patients with massive OPLL $[3,6]$. Anterior decompression and fusion (ADF) is theoretically suitable for the treatment of OPLL because it can provide direct decompression to the spinal cord and can stabilize the involved segments [4, 7]. We previously conducted a prospective study comparing ADF and posterior laminoplasty (LAMP) for the treatment of OPLL. We found that ADF is superior for neurological improvement in patients who have massive OPLL with a $\geq 50 \%$ canaloccupying ratio [8]. However, the ventral decompression procedure is complex and technically demanding, particularly in OPLL with a great occupying ratio [9].

Previous studies demonstrated that the use of threedimensional fluoroscopy is efficacious for intraoperative evaluation of bone decompression in short-segment anterior cervical discectomy and fusion (ACDF) [10]. Recently, high-resolution reconstructed computed tomography (CT) images can be obtained intraoperatively [11] and are useful for the intraoperative evaluation of adequate decompression during technically demanding anterior cervical surgeries. Here, we introduce the procedure of anterior corpectomy and fusion with the floating method for massive OPLL with the support of intraoperative CT images. Furthermore, the surgical results of this technique were compared with a historical control of OPLL cases that underwent ADF without the use of intraoperative CT.

\section{Materials and methods}

This study was approved by an institutional review board. Written informed consents for participation and for publication have been obtained from the participants. Consecutive patients treated with multi-level ADF for cervical myelopathy caused by OPLL at our institution were included. Patients with myelopathy caused by cervical disc herniation, spondylosis, or tumor, patients with a history of previous cervical spine surgery or injury, and patients who had OPLL that extended to the $\mathrm{C} 1 / \mathrm{C} 2$ level and thoracic spine with cord compression were excluded. Also, patients with OPLL treated by posterior procedure were excluded. Twenty-five OPLL patients (20 males, 5 females, average age of 63.2 years old; Table 1 ) who underwent ADF with the floating method using intraoperative CT at our institution between 2012 and 2015 were investigated in this study. The surgical results from the patients who underwent ADF using intraoperative CT (CT group) were compared with 25 consecutive patients ( 21 males, 4 females, average age of 58.8 years old; Table 1 ) who underwent ADF without using intraoperative CT before December 2011 (non-CT group).

\section{Surgical procedures}

We applied the anterior OPLL floating technique described by Yamaura et al. [7, 12] (Fig. 1a). All the surgeries were done by attending spine surgeons certified by the Japanese Society for Spine surgery and related Research. Spinal monitoring during the operation was performed in all patients. A radiolucent operating table made of carbon was used. A standard Smith-Robinson approach to the cervical spine was used [13]. After confirmation and exposure of the appropriate vertebral levels, corpectomy was performed by removing the discs and vertebral bodies. Upon reaching the posterior cortex, drilling was performed carefully under a microscope. The ossification was thinned to the shape of the bottom of a ship until part of the ossification was removed, and the soft tissue underneath the ossification first became visible or felt by the light touch of a microprobe. Before freeing the ossification from the surrounding bone tissues, we took intraoperative CT images to confirm that the width of the decompression and the thinning of the OPLL were appropriate. After careful draping, we moved the helical CT scanner gantry on a floor-embedded rail toward the operating table until the region for imaging was completely within the gantry (Fig. 2). Then, the surgeons moved to a separate room and provided slice plan instructions for scanning to the CT operator, and the cervical spine was scanned using a 16-row multidetector CT unit (TOSHIBA Medical, Tokyo, Japan). The series consisted of $0.5-\mathrm{mm}$-thick CT sections that were acquired in helical mode and were reconstructed at 0.5 $\mathrm{mm}$ intervals. The acquisition parameters were $120 \mathrm{kV}$

Table 1 Demographics

\begin{tabular}{llll}
\hline & $\begin{array}{l}\text { Non-CT group } \\
(n=25)\end{array}$ & $\begin{array}{l}\text { CT group } \\
(n=25)\end{array}$ & $P$ \\
\hline Age & $63.5 \pm 9.1$ & $58.8 \pm 11.4$ & 0.19 \\
Gender (M/F) & $20 / 5$ & $21 / 4$ & 0.71 \\
Pre JOA score & $11.6 \pm 1.7$ & $11.4 \pm 3.3$ & 0.83 \\
Canal-occupying ratio (\%) & $48.8 \pm 14.4$ & $57.8 \pm 17.0$ & 0.06 \\
Levels of fusion & $3.3 \pm .0 .8$ & $3.0 \pm 0.6$ & 0.10 \\
\hline
\end{tabular}

Mean \pm standard deviation 


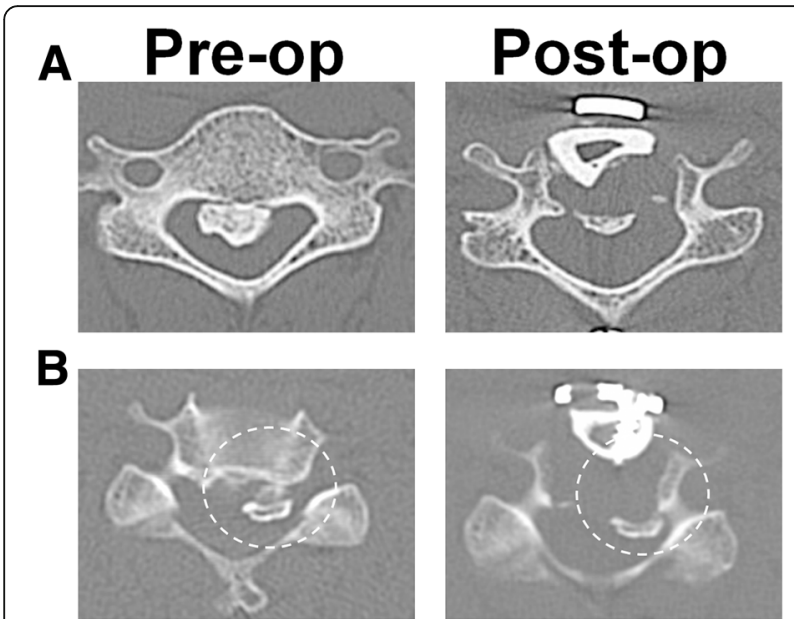

Fig. 1 a Preoperative (Pre-op) and postoperative (Post-op) \T (computed tomography) images in the axial plane showed complete floating of ossification of the posterior longitudinal ligament (OPLL). b Insufficient floating of OPLL due to the "impingement" between OPLL and medial aspect of the uncovertebral joint

and $400 \mathrm{~mA}$. After we had confirmed that the region for imaging was appropriately scanned, the CT gantry was moved away from the operating table, and the CT images were reviewed. In the axial images, we checked the decompression width, position of the vertebral artery and pedicle, approach angle, and thinning of the OPLL (Fig. 3a). In the sagittal images, we evaluated whether decompression was sufficient to reach above or below the spinal cord compression by OPLL (Fig. 3b). This process usually took $10-15 \mathrm{~min}$ from the draping to completing the review of the CT images. After review of the images, further thinning of the OPLL or removal of surrounding bone was performed as deemed necessary, to complete the floating of the OPLL. When freeing the ossification from the surrounding bone tissues, the top

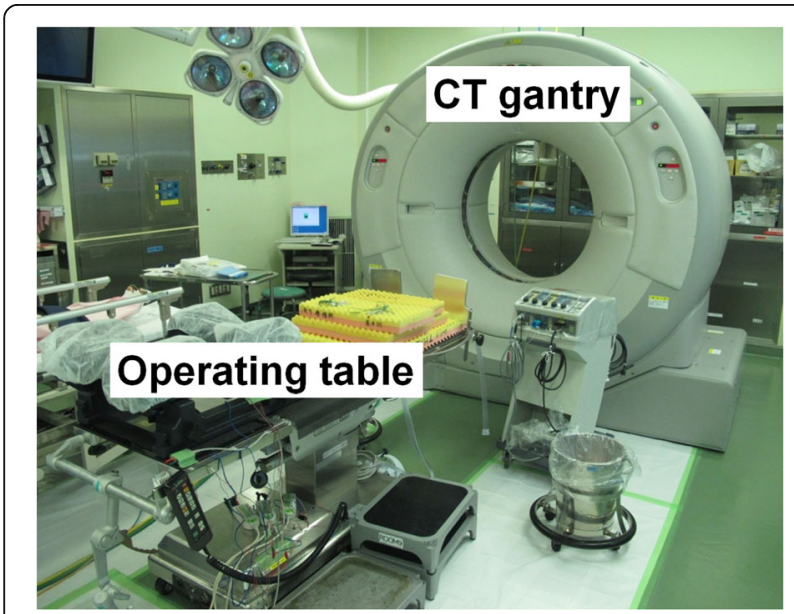

Fig. 2 A multidetector $C T$ with mobile gantry in the operating room

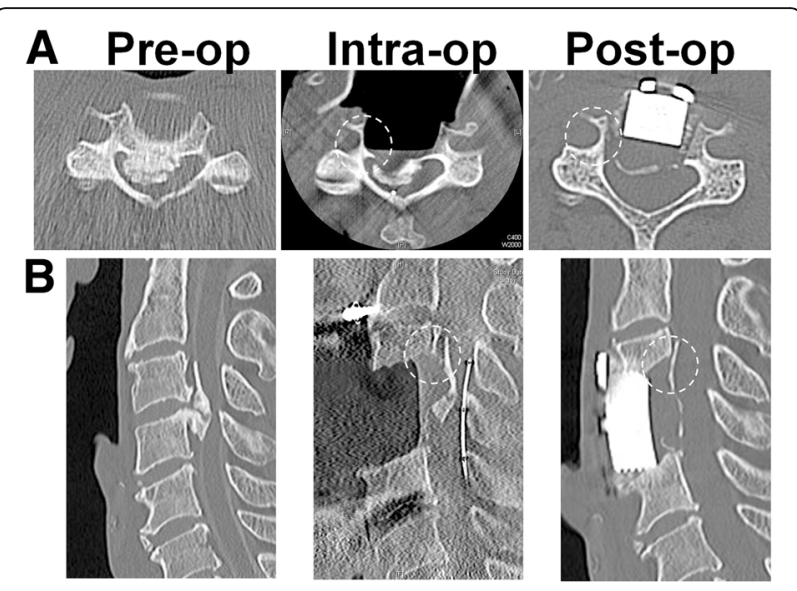

Fig. 3 Pre-op, intraoperative (Intra-op), and Post-op CT images in the axial plane (a) and sagittal plane (b)

and bottom ends of the ossification were first cut transversely and then disconnected from the pedicle that was situated laterally. When the thinned OPLL appeared to be like a board floating on water, the decompression of the entire spinal cord was complete. For reconstruction, we used a hydroxyapatite block or a fibular bone graft reinforced by an anterior plate.

\section{Clinical outcomes}

Neurological functions were evaluated using the Japanese Orthopaedics Association (JOA) scoring and associated recovery rate [8]. Additionally, the operation time, intraoperative bleeding, and postoperative complications were evaluated.

\section{Evaluations of decompression using postoperative CT images}

Postoperative CT scans were obtained within 3 months after surgery to assess the decompression using a 64-row multidetector CT unit (TOSHIBA Medical). We evaluated whether the decompression was sufficiently complete in the reconstructed CT images. If the decompression width was insufficient in the axial or sagittal plane, impingement between OPLL and the surrounding bone tissue (the posterior wall of vertebrae, the medial aspect of the pedicle or the uncovertebral joint) could occur and disturb the floating of the OPLL (Fig. 1). The patients were followed for at least 1 year postoperatively.

Statistical analyses were performed using unpaired $t$ tests for continuous variables and Fisher's exact test for categorical data. The significance level was set at $p<0.05$.

\section{Results}

Neurological disturbance was improved in all the 25 patients; the average improvement rate in JOA score was $65.7 \%$. There was no additional morbidity or progression 
of neurological symptoms in patients from CT group. Intraoperative reconstructed CT images were successfully obtained from all the patients. Scanning time was usually approximately $60 \mathrm{~s}$. The complete procedure from draping to evaluation of the pictures took less than $15 \mathrm{~min}$. All spinal segments to be decompressed were successfully visualized in axial, sagittal, and coronal views with excellent resolution.

In comparison with the non-CT group, no significant differences were found in comparisons of patient age, gender, preoperative neurological status based on JOA score, and levels of surgery (Table 1). The occupying ratio of OPLL tended to be greater in the CT group $(p=0.06)$, although no significant difference was found. There were no differences in intraoperative blood loss. However, the operating time tended to be shorter in the CT group (Table 2).

In the non-CT group, incomplete floating of the OPLL was observed for four patients $(16.0 \%)$ on the postoperative CT images (Table 2). In these patients, impingement between OPLL and the medial aspect of the pedicle or uncovertebral joint that disturbed the complete floating of the OPLL (Fig. 1b) was observed. On the other hand, insufficient decompression was not found in any patients who underwent ADF using intraoperative CT. There were no significant differences in postoperative neurological improvement rate (IR) between the CT and nonCT groups (Table 2).

Eight patients $(32.0 \%)$ presented with perioperative complications in the non-CT group, including persistent dysphasia in three patients, upper airway obstruction requiring re-intubation in one patient, dislodgement of the graft in two patients, and C5 palsy in one patient. Perioperative complications were observed in only four patients $(16.0 \%)$ in the CT group, including C5 palsy in two patients, dislodgement of the graft in one patient, and persistent dysphasia in one patient.

\section{Discussion}

The anterior procedure has been widely used for the treatment of cervical degenerative diseases. Generally, for cases in which the compressive pathology is ventral to the spinal cord, an anterior procedure is preferably applied [14, 15]. An anterior approach allows for direct removal of the

Table 2 Surgical outcomes in non-CT and CT group

\begin{tabular}{llll}
\hline & $\begin{array}{l}\text { Non-CT group } \\
(n=25)\end{array}$ & $\begin{array}{l}\text { CT group } \\
(n=25)\end{array}$ & $P$ \\
\hline Operating time (min) & $402.0 \pm 125.2$ & $335.9 \pm 97.2$ & 0.05 \\
Intra-op blood loss (g) & $461.7 .2 \pm 832.4$ & $358.0 \pm 375.6$ & 0.56 \\
Impingement (+) & $4(16.0 \%)$ & $0(0 \%)$ & 0.05 \\
Post-op JOA score & $14.9 \pm 1.5$ & $15.1 \pm 1.5$ & 0.68 \\
IR (\%) & $64.4 \pm 18.8$ & $65.7 \pm 19.8$ & 0.82 \\
\hline
\end{tabular}

Mean \pm standard deviation

$I R$ improvement rate in JOA score compressive pathology without the manipulation of the cord. Therefore, ADF is a good option for the treatment of OPLL because it can directly decompress the spinal cord by floating the OPLL and stabilizing the involved segments $[7,16]$. Along with others, we have reported that the anterior procedure is particularly efficacious for patients with massive OPLL [8].

The goals of ADF include the removal of neural compression, the restoration of stability, and the restoration or maintenance of spinal alignment [17]. Cooper et al. [18] described that the adequacy of spinal cord decompression is probably the most important factor in determining the outcome. However, anterior decompression for OPLL is sometimes technically demanding, particularly for massive OPLL, which is sometimes accompanied by extensive bleeding during the floating procedure, making visualization of the surgical site difficult. Additionally, massive OPLL often accompanies dural ossification, and thus, there is a high incidence of dural tear and cerebrospinal fluid leakage during decompression that can cause neurological deterioration. In fact, Kimura et al. [9] reported that the size of OPLL is a significant, independent risk factor for perioperative neurological complications in ADF for OPLL patients.

Previous reports indicated that key points for sufficient decompression in the anterior procedure for massive OPLL are adequate orientation, adequate width of decompression, and adequate thinning of the OPLL [7]. If the decompression width is narrow, the edge of the OPLL can impinge the surrounding bone tissue (the posterior wall of the vertebrae, medial aspect of the pedicle or uncovertebral joint), which makes the floating insufficient (Fig 1b). If the thinning of the OPLL is insufficient, floating of the OPLL can be affected by anterior bone graft. Despite these points, the width of the OPLL and the thinning of OPLL are sometimes insufficient because wide decompression and thinning of the OPLL are related to complications such as injury to the vertebral artery and dural tears [19].

In this study, we included patients with massive OPLL (canal-occupying ratio $47.2 \%$ in non-CT group and $57.8 \%$ in CT group) and found incomplete floating of the OPLL in $16.0 \%$ of patients. All of these patients showed an insufficient width of decompression, which disturbed floating of the OPLL. Although none of these patients showed neurological deterioration after surgery and none underwent revision surgery, their improvement rate of neurological score (IR $45.7 \pm 12.6 \%$ ) tended to be lower compared with patients who achieved adequate decompression (IR $66.8 \pm 19.4 \%$ ). Matsuoka et al. [7] also reported that insufficient decompression can cause poor outcomes after surgery and uneven decompression can cause a worsening of neurological symptoms in massive OPLL. We evaluated the CT images before floating the OPLL instead of after floating, 
because uneven floating of OPLL can cause neurological damage intraoperatively.

Recently, intraoperative imaging technology was developed. Three-dimensional fluoroscopy and navigation systems improve the accuracy of pedicle screw insertion [20]. Cone-beam type CT is also being increasingly used for difficult procedures, such as cervical pedicle screw placement [21]. An intraoperative CT imaging system with a self-moving helical CT scanner gantry can provide intraoperative, high-resolution three-dimensional imaging in the operating room. Since this type of "mobile" CT system was installed in the OR at our institution, we have used this system to assist in cervical surgeries. We previously reported that the evaluation of intraoperative CT images dramatically improved the accuracy of cervical pedicle screw insertion because surgeons could obtain precise orientation [11].

Similarly, intraoperative CT evaluation was effective in anterior decompression with fusion for OPLL. Since the patients in the CT group possessed massive OPLL, decompression was generally difficult because of the above-mentioned reasons. However, insufficient decompression was not found in any patients because we could obtain the precise orientation of the operation during the decompression field by evaluating intraoperative reconstructed images with high resolution. By checking the approach angles to the vertebrae, width and depth of decompression, thinning of the OPLL, and location of the vertebral artery, pedicle, and neural foramen intraoperatively, surgeons can complete decompression easily and appropriately. Navigation system may also be helpful to determine the resected margins intraoperatively. However, in the navigation system, it is difficult to know during surgery how much the surgeon has thinned the OPLL and removed the surrounding bone tissue.

Interestingly, the operating time was shorter for the CT group, although we used approximately 10-15 min for intraoperative CT scanning and the evaluation of images. A potential reason for this decreased operative time is that the surgeon is more certain of their intraoperative orientation and progress, and thus more confident to proceed in these difficult cases. We also considered intraoperative $\mathrm{CT}$ evaluation to be effective for lowering the learning curve for surgeons to perform ADF with floating method for OPLL. In this procedure, the surgeon can obtain informative feedback from intraoperative $\mathrm{CT}$ images during the completion of the OPLL floating.

Some limitations of this procedure should be noted. Although mobile CT is very useful for obtaining intraoperative high-resolution images, these systems are expensive and may not be available in small hospitals. However, cone-beam CT or three-dimensional fluoroscopy can alternatively be used. We have compared the surgical results in ADF using intraoperative $\mathrm{CT}$ with those in the historical control group. Thus, refinement of surgical technique and the benefit to the more recent (intraoperative CT) patients of the surgical learning curve may have an effect on the results. The radiation exposure (approximately $5 \mathrm{mSV}$ : 50 times greater as plane chest X-ray) to patients is another limitation of this technique. Therefore, we perform CT scanning only once during the surgery. To reduce the radiation exposure, we are considering substituting the postoperative CT scan for the intraoperative scan, as no patients in the intraoperative scan group required revision surgery.

\section{Conclusions}

Intraoperative CT scan provided useful information with high-resolution images and was effective in avoiding insufficient decompression and poor neurological outcomes in ADF with the floating method for massive OPLL. We also consider that the intraoperative $\mathrm{CT}$ imaging is helpful to provide informative feedback to surgeons performing skill-intensive surgeries such as ADF with the floating method.

\section{Abbreviations}

ACDF: Anterior cervical discectomy and fusion; ADF: Anterior decompression and fusion; CT: Computed tomography; IR: Improvement rate; JOA: Japanese Orthopaedics Association; LAMP: Laminoplasty; OPLL: Ossification of the posterior longitudinal ligament

\section{Acknowledgements}

We thank Kazumi Soma for his technical support to take intraoperative CT.

Funding

This work was supported by Japanese Health Labour Sciences Research Grant.

\section{Availability of data and materials}

The datasets generated and/or analyzed during the current study are not publicly available because it contains patients' personal information but are available from the corresponding author on reasonable request.

\section{Authors' contributions}

TY1 and AO conceived of the study and participated in its design and coordination. TY1, TH, TY2, HI, TK, KS, ME, SK, and YA performed the procedure and collected the data. All authors read and approved the final manuscript.

\section{Authors' information}

TY1 is a member of Spine surgery and Related Research, Cervical Spine Research Society, Orthopaedic Research Society.

$\mathrm{AO}$ is a Professor in the Department of Orthopaedic Surgery, Tokyo Medical and Dental University, Vice president of the Japanese Orthopaedic

Association, and Vice president of the Japanese Society for Spine surgery and Related Research.

\section{Competing interests}

The authors declare that they have no competing interests.

Consent for publication

All the patients agreed with the publication of this study.

Ethics approval and consent to participate

This study was approved by an institutional ethical committee (\#1777), and all the patients agreed to participate in this study.

\section{Author details}

${ }^{1}$ Department of Orthopaedic Surgery, Graduate School, Tokyo Medical and Dental University, 1-5-45 Yushima, Bunkyo-ku, Tokyo 113-8519, Japan. 
${ }^{2}$ Section of Regenerative Therapeutics for Spine and Spinal Cord, Graduate School, Tokyo Medical and Dental University, 1-5-45 Yushima, Bunkyo-ku, Tokyo 113-8519, Japan. ${ }^{3}$ Saiseikai Kawaguchi General Hospital, Kawaguchi, Japan. ${ }^{4}$ Tokyo Medical and Dental University Spine Group, Tokyo, Japan.

Received: 18 November 2016 Accepted: 11 January 2017

Published online: 19 January 2017

\section{References}

1. Matsunaga S, Kukita M, Hayashi K, et al. Pathogenesis of myelopathy in patients with ossification of the posterior longitudinal ligament. J Neurosurg. 2002;96:168-72

2. Matsunaga S, Sakou T. Ossification of the posterior longitudinal ligament of the cervical spine: etiology and natural history. Spine (Phila Pa 1976). 2012; 37:E309-14

3. Iwasaki M, Okuda S, Miyauchi A, et al. Surgical strategy for cervical myelopathy due to ossification of the posterior longitudinal ligament: Part 1: clinical results and limitations of laminoplasty. Spine (Phila Pa 1976). 2007;32:647-53.

4. Iwasaki M, Okuda S, Miyauchi A, et al. Surgical strategy for cervical myelopathy due to ossification of the posterior longitudinal ligament: Part 2: advantages of anterior decompression and fusion over laminoplasty. Spine (Phila Pa 1976). 2007;32:654-60.

5. Ogawa Y, Chiba K, Matsumoto M, et al. Long-term results after expansive open-door laminoplasty for the segmental-type of ossification of the posterior longitudinal ligament of the cervical spine: a comparison with nonsegmental-type lesions. J Neurosurg Spine. 2005:3:198-204.

6. Liu T, Xu W, Cheng T, Yang HL. Anterior versus posterior surgery for multilevel cervical myelopathy, which one is better? A systematic review. Eur Spine J. 2011;20:224-35.

7. Matsuoka T, Yamaura I, Kurosa Y, Nakai O, Shindo S, Shinomiya K. Long-term results of the anterior floating method for cervical myelopathy caused by ossification of the posterior longitudinal ligament. Spine (Phila Pa 1976). 2001:26:241-8

8. Sakai K, Okawa A, Takahashi M, et al. Five-year follow-up evaluation of surgical treatment for cervical myelopathy caused by ossification of the posterior longitudinal ligament: a prospective comparative study of anterior decompression and fusion with floating method versus laminoplasty. Spine (Phila Pa 1976). 2012;37:367-76.

9. Kimura A, Seichi A, Hoshino Y, et al. Perioperative complications of anterior cervical decompression with fusion in patients with ossification of the posterior longitudinal ligament: a retrospective, multi-institutional study. J Orthop Sci. 2012;17:667-72.

10. Deinsberger R, Regatschnig R, Ungersbock K. Intraoperative evaluation of bone decompression in anterior cervical spine surgery by three-dimensional fluoroscopy. Eur Spine J. 2005;14:671-6.

11. Yoshii T, Hirai T, Sakai K, Inose H, Kato T, Okawa A. Cervical pedicle screw placement using intraoperative computed tomography imaging with a mobile scanner gantry. Eur Spine J. 2016;25:1690-7.

12. Yamaura I. Anterior decompression for cervical myelopathy caused by ossification of the posterior longitudinal ligament-anterior floating method of OPLL. Nihon Seikeigeka Gakkai zasshi. 1996;70:296-310.

13. Smith GW, Robinson RA. The treatment of certain cervical-spine disorders by anterior removal of the intervertebral disc and interbody fusion. J Bone Joint Surg Am. 1958;40-A:607-24.

14. Edwards 2nd CC, Riew KD, Anderson PA, Hilibrand AS, Vaccaro AF. Cervical myelopathy. Current diagnostic and treatment strategies. Spine J. 2003;3:68-81.

15. Klineberg E. Cervical spondylotic myelopathy: a review of the evidence. Orthop Clin North Am. 2010;41:193-202.

16. Masaki Y, Yamazaki M, Okawa A, et al. An analysis of factors causing poor surgical outcome in patients with cervical myelopathy due to ossification of the posterior longitudinal ligament: anterior decompression with spinal fusion versus laminoplasty. J Spinal Disord Tech. 2007;20:7-13.

17. Bohlman HH, Emery SE, Goodfellow DB, Jones PK. Robinson anterior cervical discectomy and arthrodesis for cervical radiculopathy. Long-term follow-up of one hundred and twenty-two patients. J Bone Joint Surg Am. 1993;75: 1298-307.

18. Cooper PR. Anterior cervical vertebrectomy: tips and traps. Neurosurgery. 2001;49:1129-32

19. Fountas KN, Kapsalaki EZ, Nikolakakos LG, et al. Anterior cervical discectomy and fusion associated complications. Spine (Phila Pa 1976). 2007:32:2310-7.
20. V Rajan V, Kamath V, Shetty AP, Rajasekaran S. Iso-C3D navigation assisted pedicle screw placement in deformities of the cervical and thoracic spine. Indian J Orthop. 2010;44:163-8.

21. Ishikawa Y, Kanemura T, Yoshida G, et al. Intraoperative, full-rotation, threedimensional image (O-arm)-based navigation system for cervical pedicle screw insertion. J Neurosurg Spine. 2011;15:472-8.

\section{Submit your next manuscript to BioMed Central and we will help you at every step:}

- We accept pre-submission inquiries

- Our selector tool helps you to find the most relevant journal

- We provide round the clock customer support

- Convenient online submission

- Thorough peer review

- Inclusion in PubMed and all major indexing services

- Maximum visibility for your research

Submit your manuscript at www.biomedcentral.com/submit 